\title{
Eosinophil-derived IL-13 promotes emphysema
}

\author{
Alfred D. Doyle ${ }^{1}$, Manali Mukherjee ${ }^{2}$, William E. LeSuer ${ }^{3}$, Tyler B. Bittner ${ }^{3}$, \\ Saif M. Pasha ${ }^{3}$, Justin J. Frere (10 ${ }^{3}$, Joseph L. Neely ${ }^{3}$, Jake A. Kloeber (10 ${ }^{3}$, \\ Kelly P. Shim ${ }^{1,4}$, Sergei I. Ochkur ${ }^{3}$, Terence Ho ${ }^{2}$, Sarah Svenningsen ${ }^{2}$, \\ Benjamin L. Wright ${ }^{1,4}$, Matthew A. Rank ${ }^{1}$, James J. Lee ${ }^{3, \dagger}$, Parameswaran Nair ${ }^{2}$ \\ and Elizabeth A. Jacobsen ${ }^{3}$
}

Affiliations: ${ }^{1}$ Division of Allergy, Asthma and Clinical Immunology, Mayo Clinic Arizona, Scottsdale, AZ, USA. ${ }^{2}$ Division of Respirology, Dept of Medicine, McMaster University and St Joseph's Healthcare, Hamilton, ON, Canada. ${ }^{3}$ Division of Pulmonary Medicine, Dept of Biochemistry and Molecular Biology, Mayo Clinic Arizona, Scottsdale, AZ, USA. ‘Division of Pulmonology, Phoenix Children's Hospital, Phoenix, AZ, USA.

Correspondence: Elizabeth A. Jacobsen, Dept of Biochemistry and Molecular Biology, Mayo Clinic Collaborative Research Building, CR 02-217, Mayo Clinic Arizona, 13400 E. Shea Boulevard, Scottsdale, AZ 85259, USA. E-mail: jacobsen.elizabethamayo.edu

@ERSpublications

This study suggests that eosinophils may contribute to the emphysema observed in a subset of patients with chronic airways disease http://ow.ly/mBsz30nvDaG

Cite this article as: Doyle $\mathrm{AD}$, Mukherjee M, LeSuer WE, et al. Eosinophil-derived IL-13 promotes emphysema. Eur Respir J 2019; 53: 1801291 [https://doi.org/10.1183/13993003.01291-2018].

ABSTRACT The inflammatory responses in chronic airway diseases leading to emphysema are not fully defined. We hypothesised that lung eosinophilia contributes to airspace enlargement in a mouse model and to emphysema in patients with chronic obstructive pulmonary disease (COPD).

A transgenic mouse model of chronic type 2 pulmonary inflammation (I5/hE2) was used to examine eosinophil-dependent mechanisms leading to airspace enlargement. Human sputum samples were collected for translational studies examining eosinophilia and matrix metalloprotease (MMP)-12 levels in patients with chronic airways disease.

Airspace enlargement was identified in I5/hE2 mice and was dependent on eosinophils. Examination of I5/hE2 bronchoalveolar lavage identified elevated MMP-12, a mediator of emphysema. We showed, in vitro, that eosinophil-derived interleukin (IL)-13 promoted alveolar macrophage MMP-12 production. Airspace enlargement in I5/hE2 mice was dependent on MMP-12 and eosinophil-derived IL-4/13. Consistent with this, MMP-12 was elevated in patients with sputum eosinophilia and computed tomography evidence of emphysema, and also negatively correlated with forced expiratory volume in $1 \mathrm{~s}$.

A mouse model of chronic type 2 pulmonary inflammation exhibited airspace enlargement dependent on MMP-12 and eosinophil-derived IL-4/13. In chronic airways disease patients, lung eosinophilia was associated with elevated MMP-12 levels, which was a predictor of emphysema. These findings suggest an underappreciated mechanism by which eosinophils contribute to the pathologies associated with asthma and COPD. 


\section{Introduction}

Chronic obstructive pulmonary disease (COPD) is a chronic respiratory condition associated with cigarette smoking and characterised by irreversible airflow obstruction. It is often accompanied by the presence of airway inflammation and emphysema. COPD is among the leading causes of death worldwide and is increasing in prevalence [1]. There is no cure for COPD, but a better understanding of disease subtypes has led to improved therapeutic options [2-4]. The lung inflammatory cell infiltrate in COPD is key to disease pathogenesis. Alveolar macrophages and infiltrating neutrophils mediate destructive activities on the architecture and function of the lung in part through the release of proteases and inflammatory cytokines [5]. Despite the canonical classification of COPD as a neutrophilic disease, blood and airway eosinophilia is found in roughly a third of COPD patients [6-8]. Clinical studies suggest an association between eosinophilia and increased risk of exacerbation $[9,10]$. In an analysis of the SPIROMICS cohort, sputum eosinophil levels, in particular, correlated with increased exacerbations, worsening fixed airflow obstruction and poorer quality of life scores in COPD patients [11]. In the SPIROMICS cohort, significantly higher emphysema indices (as measured by quantitative computed tomography (CT)) were noted in patients stratified to the sputum eosinophil-high group [11].

Eosinophils produce a wide array of mediators, including toxic granule proteins (e.g. eosinophil peroxidase $(\mathrm{EPX})$ ), potent lipid derivatives (e.g. leukotrienes), and numerous cytokines and chemokines [12]. In mouse models of chronic lung inflammation, eosinophil mediators have been shown to contribute to pathologies observed in patients, including bronchoconstriction, fibrosis and mucus production [13, 14]. Eosinophil-derived interleukin (IL)-13 is of particular interest as it has been shown to contribute to all of these pathologies $[15,16]$. IL-13 mediates these activities through numerous cell types, including alveolar macrophages that play a key role in the lung remodelling associated with severe asthma and COPD.

Alveolar macrophage production of proteases, including matrix metalloproteases (MMPs), contributes to alveolar destruction in COPD [17]. MMPs are extracellular matrix-degrading enzymes. MMP-12, in particular, has been shown to be a critical mediator of alveolar destruction in a mouse model of COPD [18] and evidence points to its importance in COPD patients [17]. IL-13 signalling can polarise macrophages to an M2 ("alternatively activated") phenotype, a feature found in chronic asthma and COPD [19], and induce MMP-12 production. Notably, IL-13-stimulated mouse alveolar macrophages produce MMP-12 in vitro [20], and overproduction of IL-13 in the lungs of transgenic mice was sufficient to induce MMP-12 production and destruction of alveolar spaces [21].

We hypothesised that eosinophils are an important source of IL-13 in chronic lung disease, thereby contributing to remodelling of the airways resulting in emphysema. To explore this, we utilised a mouse model of chronic T-helper (Th) type 2 pulmonary inflammation and also analysed sputum samples from patients with asthma or COPD. Identification of the pathways by which emphysema develops/progresses may present new therapeutic targets and strategies to prevent or stabilise this chronic respiratory disease feature.

\section{Methods}

Mice

Strains of mice employed include C57BL/6J wild-type (WT) (Jackson Laboratory, Bar Harbor, ME, USA),

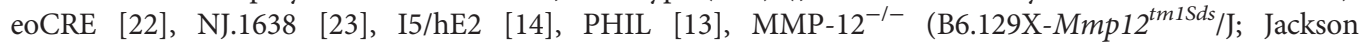

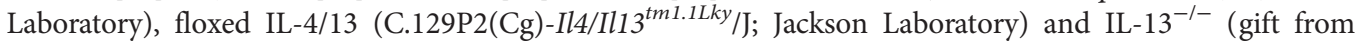
Andrew McKenzie, MRC Laboratory of Molecular Biology, Cambridge, UK [24]). Mice were analysed between 2 and 3 months of age. Mice were maintained in the Mayo Clinic Arizona Small Animal Facility (a specific pathogen-free facility) of the Mayo Clinic Arizona (Scottsdale, AZ, USA).

Studies involving mice were performed in accordance with National Institutes of Health and Mayo Clinic Institutional Animal Care and Use Committee guidelines.

\section{Cell isolation and culture}

See the supplementary material for full details.

In brief, eosinophils were isolated as described previously [25] from NJ.1638 or IL-13 ${ }^{-/-} / \mathrm{NJ} .1638$ mice $[23,24]$. IL-33 (Peprotech, Rocky Hill, NJ, USA) was added to activate the eosinophils to $50 \mathrm{ng} \cdot \mathrm{mL}^{-1}$ (IL-33 was omitted from the resting eosinophil experimental group). Cells were incubated at $37^{\circ} \mathrm{C}$ and $5 \%$ $\mathrm{CO}_{2}$, and $24 \mathrm{~h}$ later were washed 3 times to remove added cytokines. Macrophages were isolated from C57BL/6J or MMP-12 $2^{-/-}$mice as described by LASBURY et al. [26]. Cells were plated at 150000 cells per well in 24-well culture plates (Corning, Corning, NY, USA) and cultured for $48 \mathrm{~h}$ with the addition of either eosinophils (750000 cells) or media alone in $500 \mu \mathrm{L}$ total volume. To assess contact dependency, cultures were performed in transwell plates $(0.4 \mu \mathrm{m}$ pore) (Corning). 


\section{Histology}

Lung samples were processed for histological analysis as described previously [27]. Haematoxylin and eosin-stained sections were assessed for alveolar space characteristics as described previously [28].

\section{ELISA and multiplex}

MMP-12 was determined by mouse MMP-12 PicoKine ELISA (Boster Biological Technology, Pleasanton, CA, USA). EPX was measured as described previously [29]. Other mouse and human cytokines were assessed by bead-based multiplexing technology (Eve Technologies, Calgary, AB, Canada).

\section{Bronchoalveolar lavage, lung homogenate, cytospins, cell counts and differentials}

Bronchoalveolar lavage (BAL), lung collection and homogenate preparation were performed as described previously $[14,30]$. Cytospins, cell counts and differentials were performed as described previously [31, 32].

\section{Assessment of clinical sputa}

We used sputum supernatants (noncellular fraction) from 43 patients with a diagnosis of either asthma or COPD that were previously stored based on observational protocols approved by the local hospital research ethics board in St Joseph's Hospital (Hamilton, ON, Canada). These sputum samples were induced and processed as described previously [33]. Samples from asthmatic subjects were randomly selected from patients who had clinically indicated sputum testing between January and November 2017. Asthma diagnosis was based on bronchodilator reversibility and/or provocative dose causing a $20 \%$ fall in forced expiratory volume in $1 \mathrm{~s}(\mathrm{FEV} 1)<8 \mathrm{mg} \cdot \mathrm{mL}^{-1}$ upon a methacholine challenge test. The sputa of patients with COPD were collected previously as part of a local observational study during a period of clinical stability (from November 2016 to November 2017). Inclusion criteria included fixed airflow obstruction $(\mathrm{FEV} 1 / \mathrm{FVC}<0.7)$ and/or evidence of emphysema on imaging with a $\geqslant 10$ pack-year history of smoking. Those with a previous diagnosis of asthma or a significant improvement in post-bronchodilator spirometry were excluded. COPD was assessed based on Global Initiative for Chronic Obstructive Lung Disease criteria [34]. CT scans of the chest were acquired during routine clinical work-up and as such had a variety of protocols applied. The most common CT scan available was of normal resolution without contrast. Emphysema grouping was confirmed by a three-step process: 1) extrapolation from the clinical radiologist's summary report, 2) applying a quantitative CT metric (low attenuation area <950 HU (\%LAA -950 HU), using the open-source Chest Imaging Platform provided by 3D Slicer: https//: chestimagingplatform.org) and 3) confirmation of the presence of emphysema by a blinded radiologist. Eosinophilic patients were defined as those with presence of sputum eosinophils $>3 \%$ and/or evidence of blood eosinophils $>300 \mu \mathrm{L}^{-1}$, as well as those with historical evidence of eosinophils that were now suppressed due to high-dose inhaled or oral corticosteroid treatment.

\section{Statistical analyses}

Data were analysed with Prism version 7 (GraphPad, La Jolla, CA, USA). Statistical comparisons of mouse model and cell culture data were performed with the t-test. Results are represented as mean \pm SEM. Statistical comparisons between human subject groups were performed by ANOVA/Kruskal-Wallis nonparametric tests and associations were determined by Spearman's rank/Pearson correlation tests based on the distribution of the respective data sets (D'Agostino-Pearson omnibus normality tests). SPSS version 23.0 (IBM, Armonk, NY, USA) was used for multivariate regression analysis. p-values $\leqslant 0.05$ were considered significant.

\section{Results}

\section{I5/hE2 mice exhibit eosinophil-dependent airspace enlargement}

To explore the role of eosinophils in the chronically inflamed lung we employed the I5/hE2 mouse model of chronic type 2 pulmonary inflammation. The I5/hE2 mouse has been shown to develop marked airway remodelling dependent on lung eosinophilia $[14,27]$. Examination of I5/hE2 versus WT haematoxylin and eosin-stained lung sections revealed airspace enlargement in the lung parenchyma of the I5/hE2 mice (figure 1a). We crossed the I5/hE2 mouse with the eosinophil-deficient PHIL mouse [13] to examine eosinophil-dependent changes in lung structure. The cross of I5/hE2 with PHIL revealed that these lesions were entirely eosinophil dependent. Quantification of the mean intercept length between alveolar septa showed $>2$-fold increase ( 30 versus $70 \mathrm{um})$ in size in I5/hE2 mice compared with I5/hE2/PHIL mice (figure 1b).

Our previous investigations into eosinophil-dependent changes in gene expression in the I5/hE2 lung focused attention on MMP-12 as a candidate for mediating the airspace enlargement observed in I5/hE2. MMP-12 has been shown to cause the emphysema-associated breakdown of alveolar septa in a COPD model [18] and we found MMP12 was the most upregulated eosinophil-dependent gene in the I5/hE2 
a)

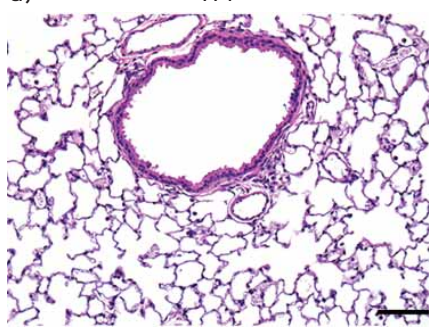

WT

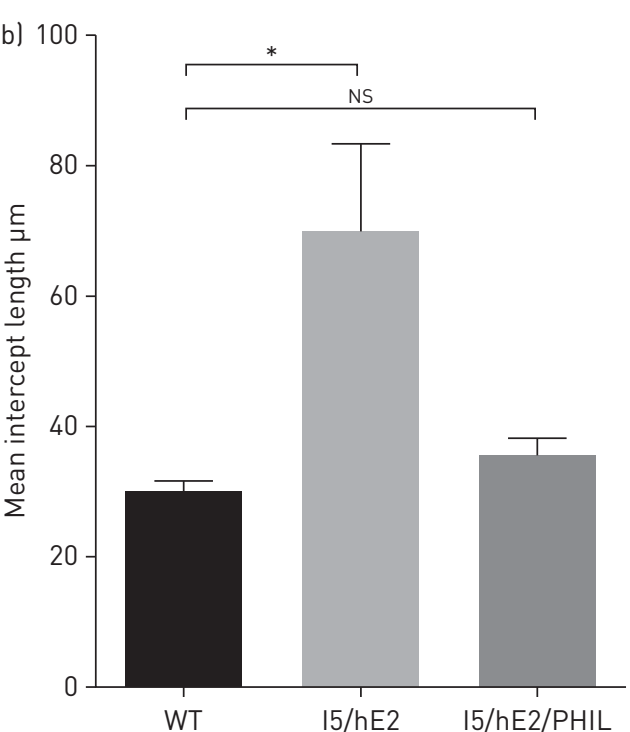

I5/hE2

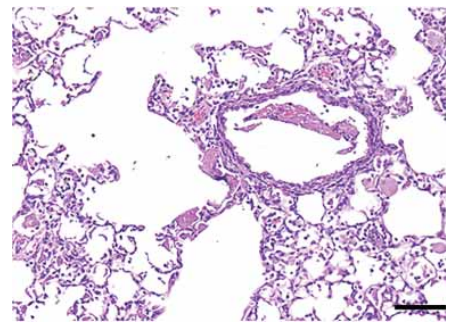

15/hE2/PHIL

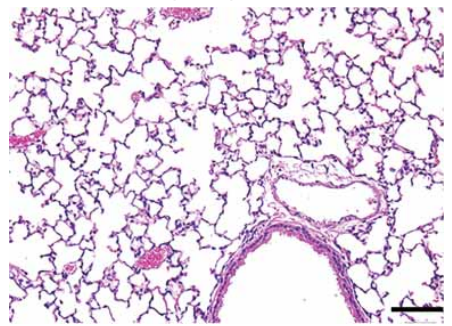

c)

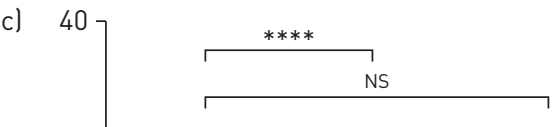

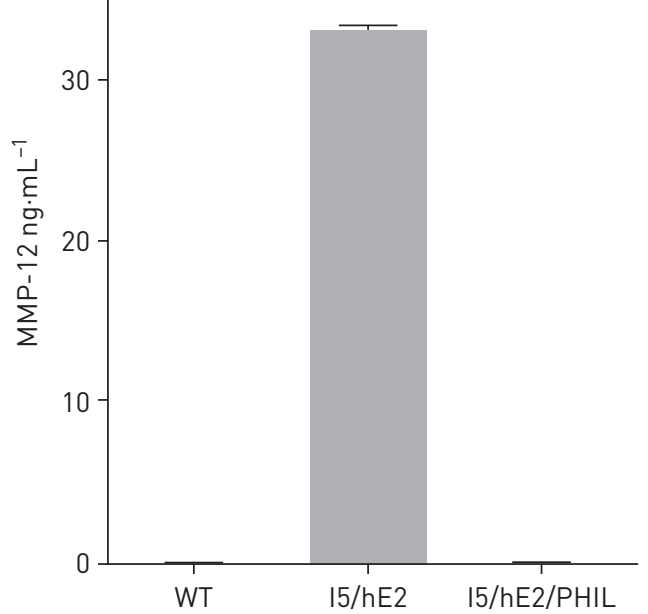

FIGURE 1 Eosinophil-dependent alveolar destruction and modulation of matrix metalloprotease (MMP)-12. a) Haematoxylin and eosin-stained lung sections demonstrate increased alveolar spaces in I5/hE2 lungs relative to wild-type (WT) and their eosinophil-deficient counterparts I5/hE2/PHIL. Lung parenchyma; scale bar: $50 \mu \mathrm{m}$. b) Quantification of average alveolar space revealed a 2-fold increase in mean intercept length in 15/hE2 lungs. $n=5$ mice. c) Bronchoalveolar lavage fluid was collected and assessed by ELISA for MMP-12, a lung remodelling mediator with dramatically increased expression in $15 / \mathrm{hE2}$ lungs. $\mathrm{n}=6 \mathrm{mice}$. *: $p<0.05 ; * * * *: p<0.0001$; Ns: nonsignificant.

lung [27]. Consistent with this observation, MMP-12 protein levels in the BAL fluid were elevated in I5/ hE2 mice and nearly undetectable in I5/hE2/PHIL mice (figure 1c). Taken together, these results suggest that eosinophils promote MMP-12 production and airspace enlargement.

MMP-12 is a mediator of alveolar destruction in I5/hE2 mice

To explore the significance of MMP-12 to the airspace enlargement observed in I5/hE2 we compared I5/ hE2 mice with their MMP-12-deficient counterparts I5/hE2/MMP-12 ${ }^{-1-}$. Histological examination of the lungs revealed reduced lesions in the absence of MMP-12 (figure 2a). Quantification of the mean intercept length between alveolar septa showed a significant reduction in I5/hE2/MMP-12 $2^{-1-}$ mice (figure $2 \mathrm{~b}$ ). However, I5/hE2/MMP-12 ${ }^{-/-}$mice had an increased mean intercept length relative to WT mice, suggesting other eosinophil-dependent mediators are contributing to these lesions. For example, other MMPs (MMP-2 and -9) have been linked with emphysema $[35,36]$ and we found these were also elevated in I5/hE2 lungs (supplementary figure S1). BAL cellularity (I5/hE2 $\left(46.85 \pm 6.45 \times 10^{5}\right.$ cells $\left.\cdot \mathrm{mL}^{-1}\right)$ and I5/hE2/ MMP-12 $2^{-/-}\left(35.23 \pm 4.25 \times 10^{5}\right.$ cells $\left.\left.\cdot \mathrm{mL}^{-1}\right)\right)$ and eosinophil numbers $\left(\mathrm{I} / \mathrm{hE2}\left(43.79 \pm 5.53 \times 10^{5} \mathrm{cells} \cdot \mathrm{mL}^{-1}\right)\right.$ and I5/hE2/MMP-12 $2^{-/-}\left(34.6 \pm 4.85 \times 10^{5}\right.$ cells $\left.\left.\cdot \mathrm{mL}^{-1}\right)\right)$ were not affected by the loss of MMP-12 in this model.

\section{Eosinophil-derived IL-13 promotes alveolar macrophage MMP-12 production in vitro}

To investigate the relationship between eosinophils and MMP-12 production we performed in vitro experiments with eosinophils and known airway MMP-12-producing cells, alveolar macrophages [37, 38]. In vitro experiments revealed that MMP-12 was not detectable in eosinophil culture supernatants, whereas MMP-12 could be detected in alveolar macrophage culture supernatants. Eosinophil and macrophage co-culture resulted in significantly increased levels of MMP-12 compared with macrophage culture 
a)

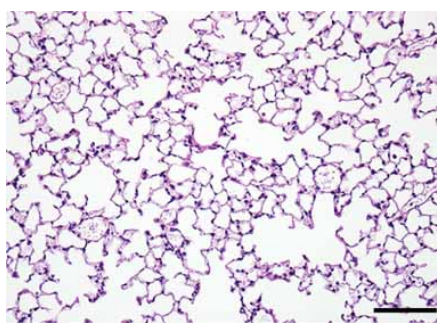

b) 100

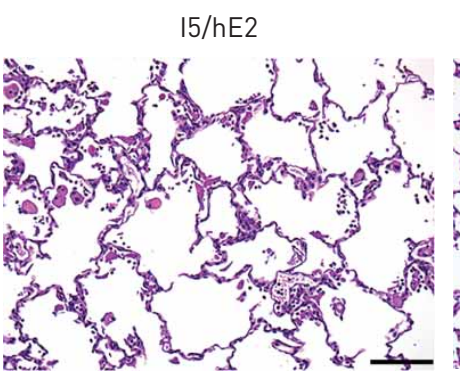

\section{5/hE2/MMP-12-/-}

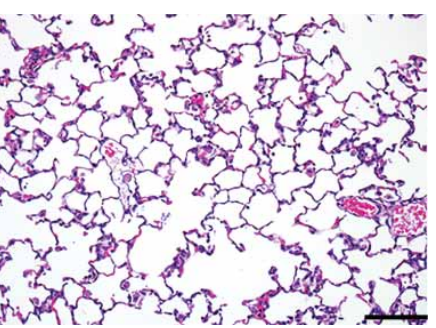

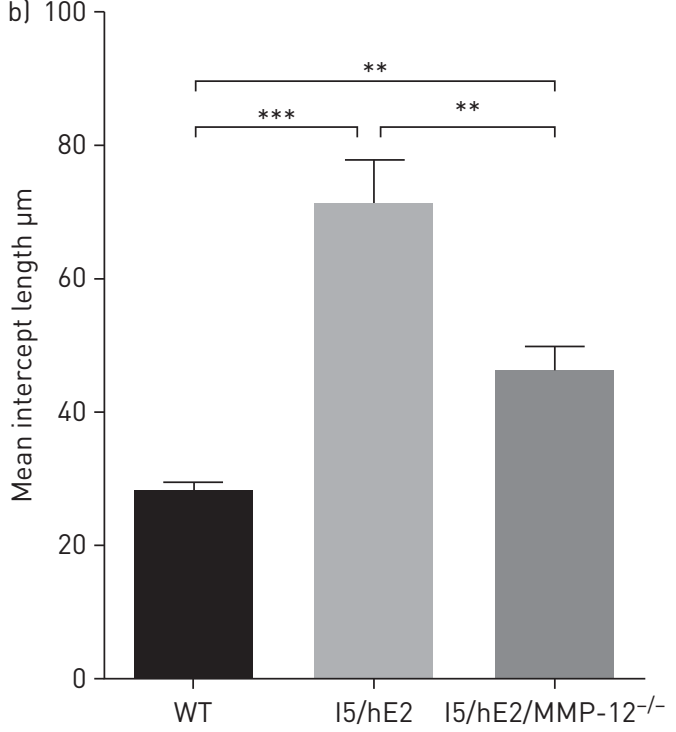

FIGURE 2 Matrix metalloprotease (MMP)-12 mediates alveolar destruction in the I5/hE2 model. a) Haematoxylin and eosin-stained lung sections demonstrate enlarged airspaces in I5/hE2 mice relative to wild-type (WT) and MMP-12 $12^{-/-}$mice. Lung parenchyma; scale bar: $50 \mu \mathrm{m}$. b) Haematoxylin and eosin-stained lung sections assessed for mean intercept length demonstrate increased alveolar spaces in $15 / \mathrm{hE2}$ mice relative to their MMP-12-deficient counterparts 15/hE2/MMP-12 ${ }^{-/-}$. $n \geqslant 3$ mice. ${ }^{* *}: p<0.01 ;{ }^{* * *}: p<0.001$.

alone (figure 3a). Co-culture of WT eosinophils with MMP-12-/- alveolar macrophages showed that the MMP-12 in these culture experiments is derived from macrophages (figure $3 \mathrm{a}$ ).

To explore the mechanism of eosinophil-mediated macrophage MMP-12 production we first assessed the role of eosinophil activation with IL-33, a cytokine associated with chronic lung disease and elevated in the I5/hE2 mouse lung $[27,39]$. Eosinophil activation by IL-33 has been demonstrated to increase release of soluble mediators that may influence the production of MMP-12 from macrophages [40, 41]. Activation of the eosinophils with IL-33 prior to co-culture with alveolar macrophages enhanced macrophage MMP-12 production relative to co-culture with nonactivated eosinophils (figure $3 \mathrm{~b}$ ). We next examined contact dependency by transwell co-culture of eosinophils with alveolar macrophages. We showed that the increased MMP-12 from macrophages is not contact dependent (figure 3c), suggesting that macrophages produce MMP-12 in response to an eosinophil-secreted mediator.

Previous studies have demonstrated IL-13 promotes MMP-12 production by macrophages [20, 36] and IL-33 is known to induce IL-13 from eosinophils $[15,42]$. We assessed the role of eosinophil-derived IL-13 by co-cultures of WT alveolar macrophages with eosinophils sufficient or deficient for IL-13. We found that eosinophil-derived IL-13 promoted alveolar macrophage MMP-12 production (figure 3d).

\section{I5/hE2 mice exhibit airspace enlargement dependent on eosinophil-derived IL-13 and MMP-12}

To examine if the lung eosinophilia in our mouse model of chronic type 2 pulmonary inflammation induces alveolar destruction via IL-13-mediated MMP-12 production we generated conditional knockout mice [43] by crossing the eosinophil-specific Cre recombinase mouse, eoCRE, with the available floxed IL-4/13 mouse [44], resulting in eosinophil-specific loss of IL-13 expression (as well as IL-4) (supplementary figure S2). We then crossed these mice with $\mathrm{I} 5 / \mathrm{hE} 2$ to create I5/hE2/eoCRE/4/13 $3^{\text {fl/fl }}$, which 

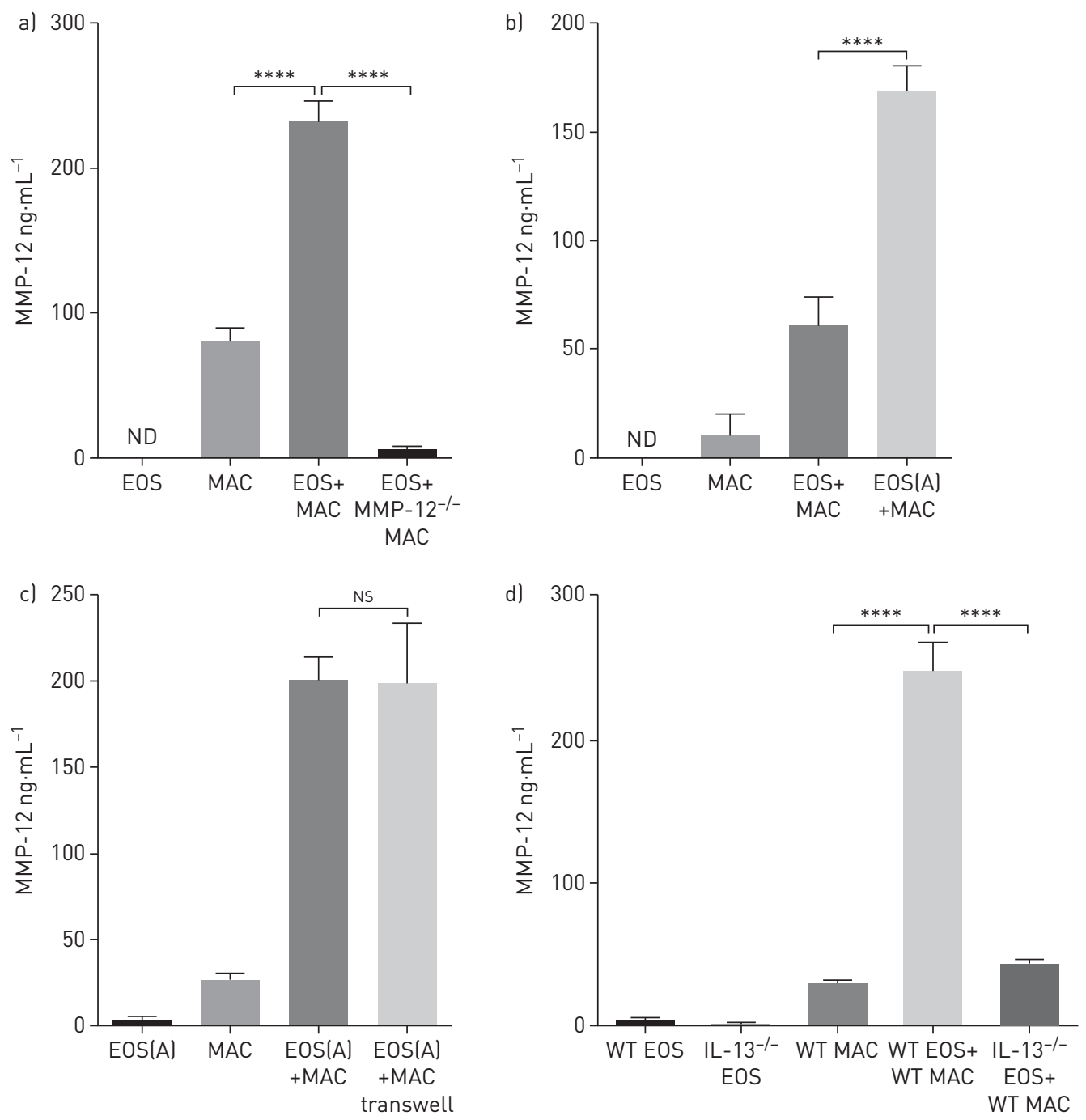

FIGURE 3 Eosinophils directly modulate alveolar macrophage production of matrix metalloprotease (MMP)-12 through interleukin (IL)-13-dependent mechanisms: ELISA measurement of MMP-12 in cell culture supernatant from in vitro $48 \mathrm{~h}$ cultures of eosinophils and/or alveolar macrophages. ND: not detected; EOS: eosinophils; MAC: macrophages; EOS(A): activated eosinophils; WT: wild-type. a) Eosinophils cultured alone, macrophages cultured alone and co-culture leosinophils+macrophages). WT eosinophils were activated (IL-33 for $24 \mathrm{~h}$ then washed) and cultured with WT macrophages or MMP-12-I- macrophages showing the MMP-12 is from macrophages. b) Assessment of co-culture supernatants from resting eosinophils versus activated eosinophils with macrophages demonstrated that pre-activation of eosinophils (IL-33 for $24 \mathrm{~h}$ then washed) enhances macrophage MMP-12 production. c) Transwell co-culture of activated eosinophils with macrophages shows that contact is not required for the enhanced macrophage MMP-12 production. d) ELISA measurements from co-culture supernatants of $\mathrm{IL}-13^{-/-}$versus WT eosinophils with WT macrophages demonstrates that eosinophil-derived IL-13 is promoting macrophage MMP-12 production. ${ }^{* * * *}$ : $p<0.0001$; NS: nonsignificant.

was compared with I5/hE2/eoCRE as a control. Histological examination of the lungs again showed airspace enlargement in I5/hE2/eoCRE that was dependent upon eosinophil-derived IL-4/13 (figure 4a). Notably, alveolar spaces in $\mathrm{I} / \mathrm{hE} 2 / \mathrm{eoCRE} / 4 / 13^{f l / f l}$ were equivalent to those of WT mice, suggesting that the enlarged alveolar spaces in I5/hE2 mice were entirely dependent on eosinophil-derived IL-4/13 (figure 4b). BAL cellularity (I5/hE2/eoCRE $\left(39.1 \pm 8.74 \times 10^{5}\right.$ cells $\cdot \mathrm{mL}^{-1}$ ) and $\mathrm{I} / \mathrm{hE} 2 / \mathrm{eoCRE} / 4 / 13^{f l / f l}$ $\left(22.98 \pm 4.31 \times 10^{5}\right.$ cells $\left.\left.\cdot \mathrm{mL}^{-1}\right)\right)$ and eosinophil numbers (I5/hE2/eoCRE $\left(26.1 \pm 4.04 \times 10^{5} \mathrm{cells} \cdot \mathrm{mL}^{-1}\right)$ and I5/hE2/eoCRE/4/13 $3^{f l f l}\left(41.53 \pm 8.28 \times 10^{5}\right.$ cells $\left.\left.\cdot \mathrm{mL}^{-1}\right)\right)$ were not affected by the loss of eosinophil-derived IL-4/13 in this model. Significantly, BAL MMP-12 levels were entirely dependent on eosinophil-derived IL-4/13 (figure 4c). Taken together, our data suggest eosinophil-derived IL-13 is a key mediator of alveolar macrophage MMP-12 production and alveolar destruction in this chronic pulmonary inflammation model. 

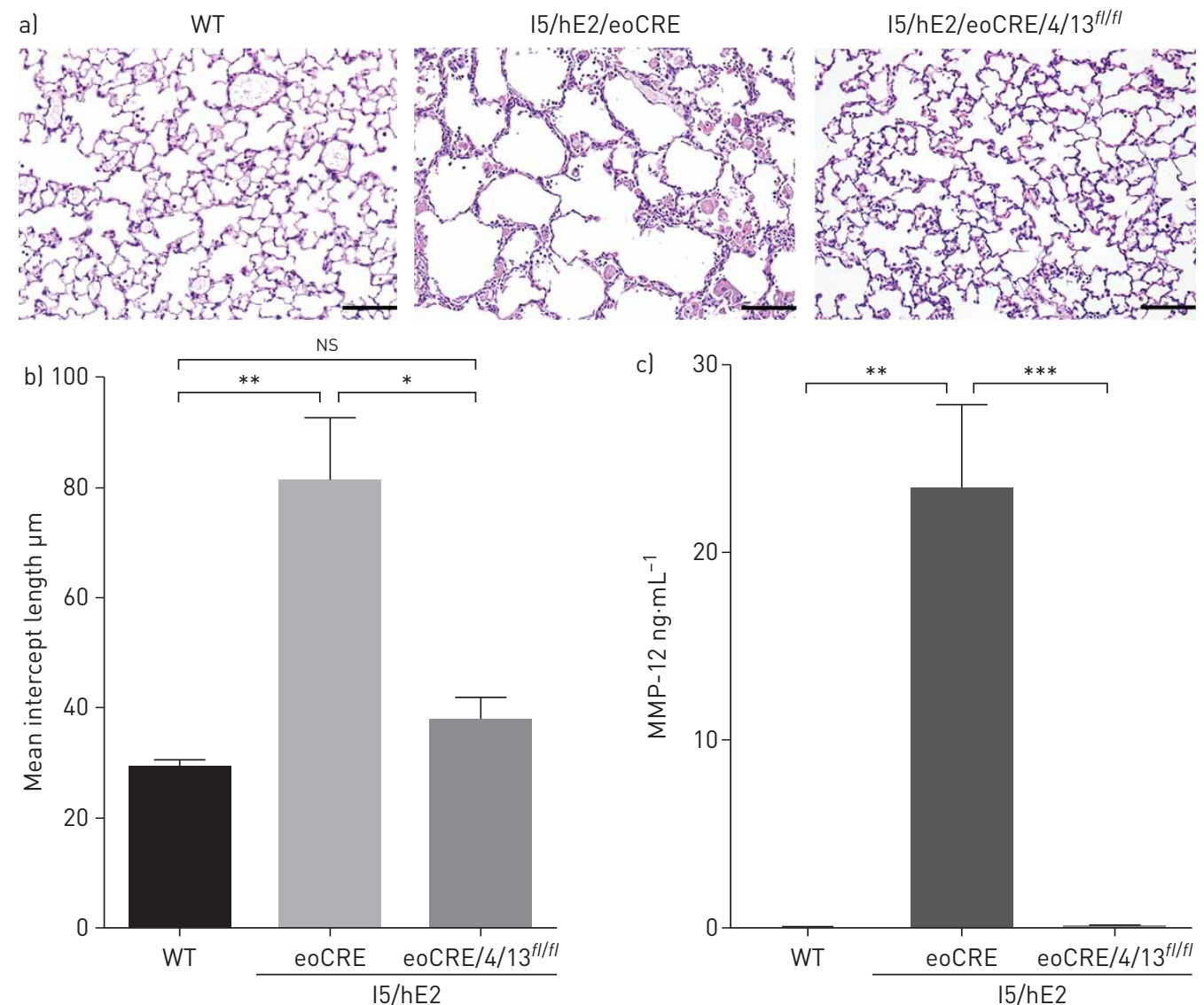

FIGURE 4 Eosinophil-derived interleukin (IL)-4/13 induces matrix metalloprotease (MMP)-12 production and alveolar destruction in I5/hE2 mice. WT: wild-type. a) A genetic cross of I5/hE2 with the eosinophil-specific Cre mouse (eoCRE) and then the available floxed $\mid \mathrm{L}-4 / 13$ mouse $\left(I 5 / \mathrm{hE} 2 /\right.$ eoCRE/4/1 $\left.{ }^{f l / f l}\right)$ revealed that enlarged alveolar spaces in I5/hE2 mice are dependent on eosinophil-derived IL-4/13. Lung parenchyma; scale bar: $50 \mu \mathrm{m}$. b) Haematoxylin and eosin-stained lung sections demonstrate enlarged airspaces in $15 / \mathrm{hE2}$ mice relative to eosinophil-derived IL-4/13-deficient mice. c) ELISA measurement of MMP-12 in bronchoalveolar lavage showed MMP-12 in I5/hE2 mice is dependent on eosinophil-derived IL-4/13. $n \geqslant 3$ mice. ${ }^{*}$ : $p<0.05 ;{ }^{* *}: p<0.01 ;{ }^{* *}: p<0.001$; Ns: nonsignificant.

\section{Elevated MMP-12 is associated with eosinophilia, emphysema and decreased lung function in human subjects}

Next, we investigated the relationship between MMP-12, eosinophilia, emphysema and lung function in humans. Out of the 44 patient sputa randomly selected, five were rejected (three rejected for comorbidities/other inflammatory abnormalities, one rejected for not meeting criteria for asthma or COPD and one rejected for age $<18$ years). Of the remaining subjects, 16 had asthma and 23 had COPD; their clinical characteristics are represented in tables 1 and 2. Sputum EPX levels were higher in the eosinophilic group $(\mathrm{p}=0.002)$ (figure $5 \mathrm{a})$ and correlated significantly with sputum IL-13 levels $(\mathrm{r}=0.536$; $\mathrm{p}=0.0004$, Spearman rank). Irrespective of diagnosis, the MMP-12 levels detected in the sputa were higher in the eosinophilic group ( $\mathrm{p}=0.01)$ (figure $5 \mathrm{~b}$ ) and modestly correlated with sputum IL-13 levels ( $\mathrm{r}=0.35$; $\mathrm{p}=0.03$ ) (figure $5 \mathrm{c}$ ). The significant correlation of MMP-12 with airway IL-13 levels and not EPX ( $\mathrm{r}=0.05$; $\mathrm{p}=0.7$ ) suggested the possibility that MMP-12 was not directly associated with eosinophilia per se, but instead associated with eosinophil-derived, and potentially other sources of, IL-13 inflammation.

In the subgroup analysis, differential expression of MMP-12 in the asthma subset with respect to eosinophilia was unremarkable $(\mathrm{p}>0.99$ ) (figure $5 \mathrm{~d}$ ); however, increased detection of MMP-12 was evident in the eosinophilic COPD airways $(\mathrm{p}=0.05)$ (figure $5 \mathrm{~d}$ ). In fact, MMP-12 levels in COPD patients were higher in those with concomitant eosinophilia and emphysema compared with those who did not exhibit either ( $p=0.02$ ) (figure $5 \mathrm{e}$ ). Comparing \%LAA $-950 \mathrm{HU}$ by eosinophilic status revealed a higher value in those with eosinophilia (median $1.4 \%$ versus $0.08 \%$; $\mathrm{p}=0.04$, Mann-Whitney test). Emphysema grouping was confirmed by the three steps described in the Methods section. The accuracy of grouping (by clinical radiologist report) was supported by a significantly higher \%LAA $-950 \mathrm{HU}$ in the emphysema group 
TABLE 1 Demographics of recruited patients: asthma and chronic obstructive pulmonary disease (COPD)

\begin{tabular}{|c|c|c|}
\hline & Asthma & COPD \\
\hline Patients & 16 & 23 \\
\hline Age years & $62 \pm 10$ & $64 \pm 11$ \\
\hline Female & $9(56)$ & $10(43)$ \\
\hline FEV $1 \%$ pred & $65 \pm 17^{*}$ & $49 \pm 17^{*}$ \\
\hline FEV $1 / F V C$ & $0.62 \pm 0.13^{*}$ & $0.49 \pm 0.13^{*}$ \\
\hline DLco \% pred & $99 \pm 33^{*, \#}$ & $61 \pm 18^{* .9}$ \\
\hline Current smoker & $1(6)^{*}$ & $12(52)^{*}$ \\
\hline Ex-smoker & $0(0)$ & 7 (30) \\
\hline Smoking history pack-years & NA & $46 \pm 24$ \\
\hline Emphysema & $1(6)^{*}$ & $15(65)^{*}$ \\
\hline Centrilobular & 1 & 7 \\
\hline Paraseptal & 0 & 1 \\
\hline Both centrilobular and paraseptal & 0 & 7 \\
\hline Eosinophilia & $11(69)$ & $11(48)$ \\
\hline Sputum eosinophils \% & $7.0 \pm 9.1$ & $5.9 \pm 15$ \\
\hline Sputum EPX $\mathrm{ng} \cdot \mathrm{mL}^{-1} \cdot \mathrm{g}^{-1}$ & $375 \pm 587 *$ & $91.4 \pm 144^{*}$ \\
\hline ICS dose $\mu \mathrm{g} \cdot$ day $^{-1}$ fluticasone equivalent & $750(2000-0)$ & $750(3500-0)$ \\
\hline ICS use & $15(94)$ & $20(87)$ \\
\hline Maintenance OCS use & $5(31)$ & $2(9)$ \\
\hline LABA use & $15(94)$ & 18 (83) \\
\hline LAMA use & $0(0)^{*}$ & $12(52)^{*}$ \\
\hline
\end{tabular}

Data are presented as $\mathrm{n}$, mean $\pm \mathrm{SD}, \mathrm{n}(\%)$ or median (minimum-maximum). All COPD patients were current smokers or ex-smokers with $\geqslant 10$ pack-year history. FEV1: forced expiratory volume in $1 \mathrm{~s}$; FVC: forced vital capacity; DLCo: diffusing capacity of the lung for carbon monoxide; NA: not available; EPX: eosinophil peroxidase; ICS: inhaled corticosteroid; OCS: oral corticosteroid; LABA: long-acting $\beta$-agonist; LAMA: long-acting muscarinic antagonist. ${ }^{\#}$ : based on $\mathrm{n}=3$; ${ }^{\text {१: }}$ : based on $\mathrm{n}=9$. *: statistically significant difference between groups $(p \leqslant 0.05)$.

TABLE 2 Demographics of recruited patients: eosinophilic and noneosinophilic

\begin{tabular}{|c|c|c|}
\hline & Eosinophilic & Noneosinophilic \\
\hline Patients & 22 & 17 \\
\hline Asthma & $11(50)$ & $5(29)$ \\
\hline Age years & $63 \pm 7.5$ & $64 \pm 14$ \\
\hline Female & $10(46)$ & 9 (53) \\
\hline FEV $1 \%$ pred & $52 \pm 18$ & $60 \pm 19$ \\
\hline FEV $1 / F V C$ & $0.53 \pm 0.15$ & $0.56 \pm 0.14$ \\
\hline DLco \% pred & $71 \pm 32^{\#}$ & NA \\
\hline Current smoker & 5 (23) & 9 (53) \\
\hline Ex-smoker & $5(23)$ & $2(12)$ \\
\hline Smoking history pack-years & $25 \pm 32$ & $42 \pm 24$ \\
\hline Emphysema & $8(36)$ & $8(47)$ \\
\hline Centrilobular & 5 & 3 \\
\hline Paraseptal & 1 & 0 \\
\hline Both centrilobular and paraseptal & 2 & 5 \\
\hline Sputum eosinophils $\%$ & $11 \pm 15^{*}$ & $0.25 \pm 0.40^{*}$ \\
\hline Sputum EPX $\mathrm{ng} \cdot \mathrm{mL}^{-1} \cdot \mathrm{g}^{-1}$ & $293 \pm 506^{*}$ & $81 \pm 118^{*}$ \\
\hline ICS dose $\mu \mathrm{g}$-day ${ }^{-1}$ fluticasone equivalent & $750(2000-0)$ & $500(3500-0)$ \\
\hline ICS use & $19(86)$ & $16(94)$ \\
\hline Maintenance OCS use & $5(23)$ & $2(12)$ \\
\hline LABA use & $20(91)$ & $14(82)$ \\
\hline LAMA use & $7(32)$ & $5(29)$ \\
\hline
\end{tabular}

Data are presented as $\mathrm{n}, \mathrm{n}(\%)$, mean \pm SD or median (minimum-maximum). FEV1: forced expiratory volume in $1 \mathrm{~s}$; FVC: forced vital capacity; DLCO: diffusing capacity of the lung for carbon monoxide; NA: not available; EPX: eosinophil peroxidase; ICS: inhaled corticosteroid; OCS: oral corticosteroid; LABA: long-acting $\beta$-agonist; LAMA: long-acting muscarinic antagonist. \#: based on $n=7$. *: statistically significant difference between groups $(p \leqslant 0.05)$. 

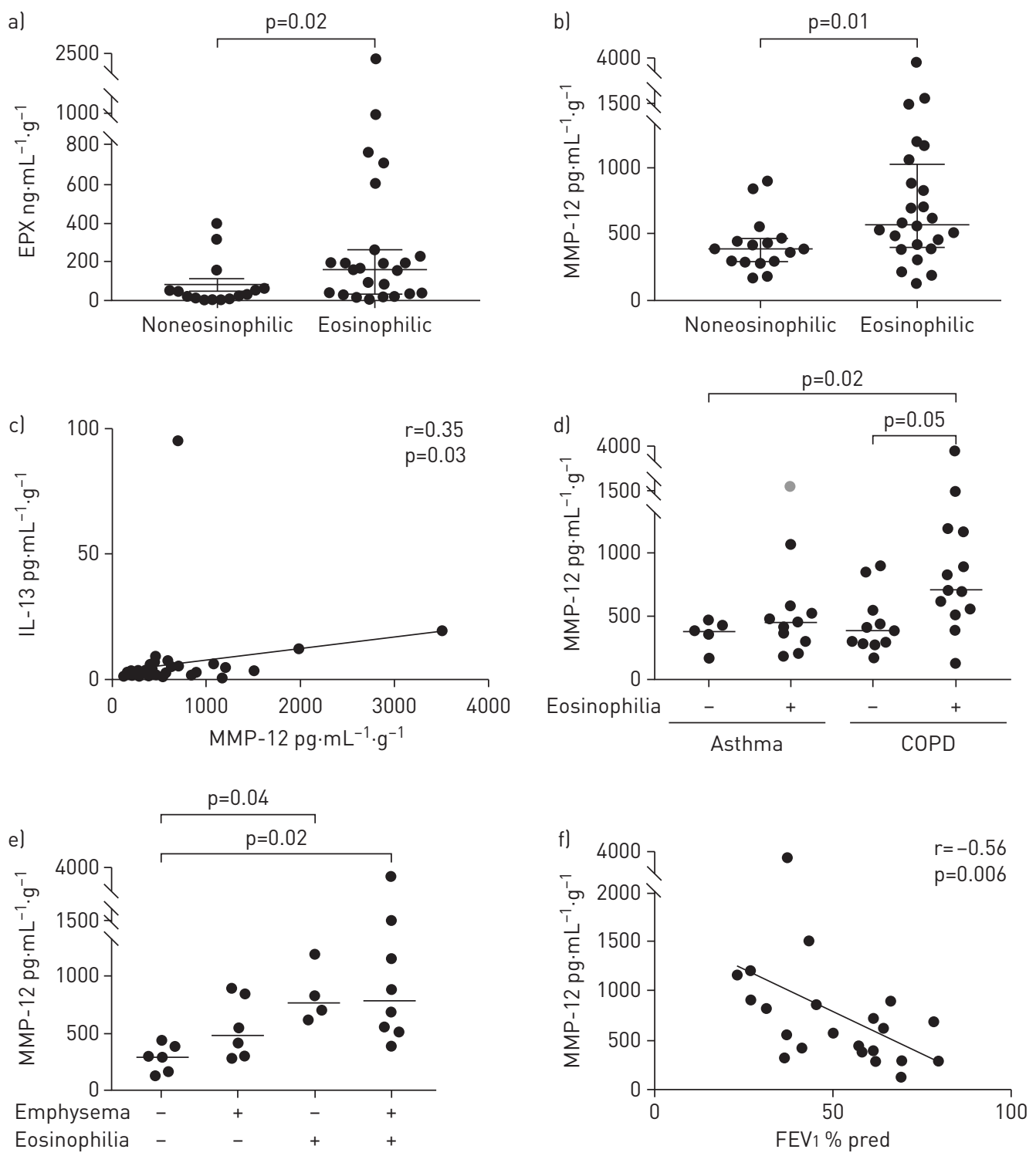

FIGURE 5 Matrix metalloprotease (MMP)-12 expression in eosinophilic emphysematous airways. EPX: eosinophil peroxidase; IL: interleukin; FEV1: forced expiratory volume in 1 s; COPD: chronic obstructive pulmonary disease. a) EPX and b) MMP-12 expression in airways of $n=40$ patients with chronic airways disease (diagnosis of either asthma or COPD) classified based on eosinophilia. Mann-Whitney test. c) Correlation of sputum MMP-12 with IL-13 $(n=40)$. Spearman rank. d, e) MMP-12 expression in airways of d) both asthma ( $n=16)$ and COPD ( $n=24)$ subjects, classified based on eosinophilia, and e) COPD subjects only, classified with respect to eosinophilia and emphysema. Kruskal-Wallis with Dunn's multiple correction. f) Correlation of sputum MMP-12 with FEV $1 \%$ pred in COPD patients ( $n=24)$. Spearman rank. Each symbol represents individual patient data and, where appropriate, the median is indicated for each subset. The grey symbol in (d) indicates an asthmatic patient with evidence of airspace enlargement. $p \leqslant 0.05$ considered as significant.

(median $1.97 \%$ versus $0.18 \% ; \mathrm{p}=0.0073$, Mann-Whitney test) and confirmation of emphysema by a blinded radiologist for each subject. Of interest, the patient in the eosinophilic asthma group who had the highest level of MMP-12 (marked as the grey symbol in figure 5d) had CT evidence of air space enlargement. If this data-point is removed, MMP-12 levels were significantly higher in the eosinophilic COPD patient sputa compared with eosinophilic asthmatic subjects ( $\mathrm{p}=0.01$, Mann-Whitney), which were otherwise comparable ( $p=0.43$ ) (figure $5 \mathrm{~d}$ ). In a multivariate regression model, MMP-12 was computed to be a predictor $\left(\beta=0.361, R^{2}=0.13\right.$, standard error of estimate $\left.0.47 ; p=0.02\right)$ for the presence of emphysema (CT evidence) and not eosinophilia, EPX or IL-13 (supplementary table S1). Finally, MMP-12 levels were negatively associated with FEV1 \% pred in patients with COPD ( $r=-0.56 ; \mathrm{p}=0.006)$ (figure 5f) and this association remained significant with the inclusion of asthmatic participants $(r=-0.45 ; p=0.005)$. 


\section{Discussion}

Although type 2 inflammation is more frequently associated with asthma than COPD, it can be present or absent in either condition. In this study, we used a mouse model of chronic type 2 pulmonary inflammation (I5/hE2) to explore the link between eosinophils and alveolar destruction, without any connotation regarding the physiological features required for a diagnosis of asthma or COPD, or involvement of smoking-induced pathways. These mice develop eosinophil-dependent lung pathologies that are absent when crossed with eosinophil-deficient mice (I5/hE2/PHIL), but may be restored with eosinophil-sufficient bone marrow engraftment [14]. We found that I5/hE2 mice develop eosinophil-dependent airspace enlargement. Significantly, MMP-12, a protease known to be a critical mediator of alveolar destruction, was elevated in I5/hE2 lungs and this was entirely dependent upon the presence of eosinophils. We demonstrated ex vivo that eosinophil-derived IL-13 stimulated alveolar macrophages to produce MMP-12. To confirm these pathways in vivo we crossed I5/hE2 with various genetically engineered mice and showed that eosinophils promote airspace enlargement dependent on IL-13 (and possibly IL-4, as we utilised the available floxed IL-4/13 mouse) and MMP-12. Consistent with these observations, it has been shown previously that MMP-12 is induced by IL-13 in the lung [20, 36] and eosinophils are a known source of IL-13 [15, 42]. Furthermore, overexpression of IL-13 in the mouse lung resulted in increased MMP-12 levels and airspace enlargement [36]. Taken together, these data reveal a clear path by which eosinophils may contribute to the destruction of airspaces in chronic respiratory diseases.

Eosinophil-derived IL-13 has been demonstrated to promote M2 macrophage differentiation as well as accumulation in a mouse model of allergic pulmonary inflammation [15]. M2 alveolar macrophages have been identified as MMP-12-producing cells (reviewed in [38]). Notably, we found that other proteases associated with alveolar destruction, i.e. MMP-2 and -9, are also elevated in the lungs of I5/hE2 mice dependent on eosinophil-derived IL-4/13. Eosinophils have been shown to express MMP-9 in asthmatic airways [45] and it is likely that eosinophils are indirectly linked, through interactions with other cell types such as macrophages, to the production of numerous remodelling agents, including MMP-2 and -9 . Indeed, we showed that removal of MMP-12 only partially reduced the alveolar destruction in I5/hE2 lungs and observed that in older MMP-12-deficient I5/hE2 mice the extent of alveolar destruction approached that of I5/hE2 (data not shown), suggesting a role for other mediators. Key contributors likely include other MMPs and cathepsins previously observed to contribute to these changes in a transgenic mouse with overexpression of IL-13 in the lung [21]. Future studies are needed to determine other key eosinophil-associated mediator(s) of alveolar destruction in the I5/hE2 lung. Taken together, these interactions highlight the role of eosinophils as regulators of local immune responses and remodelling events [46].

The I5/hE2 model enabled us to isolate the contribution of eosinophils to airspace enlargement. It is likely that other cell types can participate in the pathways explored in this work. In particular, group 2 innate lymphoid cells (ILC2s) and Th2 T-cells produce IL-13 in the lung (reviewed in [47, 48]); moreover, the airway epithelium and smooth muscle have been identified as producers of MMP-12 [49, 50]. Nevertheless, our finding that eosinophils can mediate these activities is intriguing. While IL-13 is a secreted factor, it is possible that eosinophils are a particularly potent source for these interactions. Clearly, the interactions between eosinophils and alveolar macrophages deserve further investigation as it may be possible that a spatial, temporal and/or multifactor association (e.g. IL-13 in combination with other signals) is important for these activities.

A pathogenic role of eosinophils in COPD has long been considered. Despite their potential importance in the pathophysiology of COPD, efforts to target eosinophils in clinical trials of COPD have met with limited success. A very modest clinical benefit has been noted in patients with blood eosinophilia post-treatment with subcutaneous mepolizumab (an anti-IL-5 antibody that results in eosinophil depletion) (METREX trial) [51]. In the parallel study evaluating two doses of mepolizumab as an add-on treatment for frequently exacerbating COPD patients characterised by eosinophil level (METREO trial), the annual rate of exacerbations compared with the placebo arm was deemed insignificant [51]. These findings corroborated those reported in a smaller study where the drug reduced both sputum and blood eosinophils, and yet failed to show any significant improvement in the rate of exacerbations, lung function or radiological evidence of disease severity [52]. Similarly, targeting eosinophils in COPD using benralizumab (an anti-IL-5 receptor $\alpha$ antibody that results in eosinophil depletion) showed no clinical improvement in the rate of exacerbations compared with placebo. However, a modest improvement in FEV1 was reported in the drug arm, indicating eosinophils or, by extension, eosinophilic factors might be affecting lung function [53].

The limited improvement in clinical trials examining anti-eosinophil agents in COPD may be due to irreversible airspace enlargement. Our experiments suggest a role of eosinophils in the initiation/ development of airspace enlargement, albeit indirectly through stimulation of MMP-12 (and possibly other 
protease) production by alveolar macrophages. Indeed, in COPD patients with evidence of eosinophilia there was a detectable increase in MMP-12, which was computed to be a predictor for the presence of CT evidence of emphysema. These findings suggest strategies targeting eosinophils in eosinophilic COPD may be beneficial, but that long-term studies with lung function as the primary outcome may be needed to appreciate this benefit. Alternative strategies to IL-5 blockade that target eosinophils and other cells (e.g. ILC2s) could potentially reduce exacerbations, and should be considered for future studies. One such strategy suggested by previous observations and the findings from this study is a combination of IL-33 and IL-13 blockade.

The concept of eosinophil-driven emphysema has been studied previously within COPD cohorts, which identified associations between blood eosinophils and markers of connective tissue destruction [54], and between sputum eosinophils and emphysema indices on quantitative CT [11]. Furthermore, a network analysis incorporating measures of the blood, bone marrow, as well as clinical variables, demonstrated that the capacity for immune-mediated repair was impaired in the presence of elevated blood eosinophils and/ or emphysema [55]. The current study provides mechanistic, mouse-model evidence and corroborating data from a "real-life" COPD cohort that lend support to the previous findings.

In summary, our findings reveal a role for eosinophil-derived IL-13 in alveolar destruction through induction of MMP-12. Asthma and COPD are diagnosed based on physiological criteria, with the former demonstrating reversible airflow obstruction or airway hyperresponsiveness, and the latter demonstrating fixed airflow obstruction. We translated our findings to patients with eosinophilic COPD, suggesting a contribution of eosinophil-mediated induction of MMP-12 in the development of emphysema. Sputa was tested in patients with asthma or COPD, given that eosinophilic inflammation can be a shared component between these diseases, but emphysema is not classically associated with asthma. It is interesting to speculate that our findings may extend to other chronic inflammatory respiratory diseases. Notably, asthma disease progression and severity is associated with eosinophilia and may develop into/overlap with COPD. Indeed, the asthmatic patient with highest MMP-12 detection (levels comparable to the COPD subset) had persistent eosinophilia as well as CT evidence of emphysema. A recent report from GelB et al. [56] builds on evidence of asthma subjects with a type 2 phenotype developing mild emphysema and loss of lung elasticity. In addition, a case report of a nonsmoking eosinophilic bronchitis patient developing emphysema highlights this possibility [57]. Taken together, these findings suggest that a focus on therapeutic management of lung eosinophilia, IL-13 and proteases including MMP-12 may provide effective strategies for slowing the progression of emphysema in certain patient populations.

Acknowledgements: We thank all members of the Lee laboratory group (Mayo Clinic Arizona, Scottsdale, AZ, USA) for their support, and we recognise the excellent administrative support provided by Stefanie Brendle, Linda Mardel and Shirley "Charlie" Kern (Mayo Clinic Arizona). We also recognise Melanie Kjarsgaard, Nicola LaVigne, Katherine Radford and Colm Boylan (McMaster University and St Joseph's Healthcare, Hamilton, ON, Canada) for their expert technical assistance. This work is dedicated to the memory of our dear friend and colleague James "Jamie" Lee whose larger than life character was matched only by his scientific genius. We miss him dearly.

Author contributions: A.D. Doyle, J.J. Frere and J.J. Lee designed the research study. A.D. Doyle, W.E. LeSuer, T.B. Bittner, S.M. Pasha, J.J. Frere, J.L. Neely, J.A. Kloeber, K.P. Shim, S.I. Ochkur, T. Ho and M. Mukherjee performed the research. A.D. Doyle, W.E. LeSuer, T.B. Bittner, S.M. Pasha, J.J. Frere, S.I. Ochkur, B.L. Wright, M.A. Rank, M. Mukherjee, P. Nair, J.J. Lee and E.A. Jacobsen analysed the data. A.D. Doyle wrote the initial draft of the manuscript. P. Nair, B.L. Wright, M.A. Rank, M. Mukherjee and E.A. Jacobsen provided critical assessments during the revision process leading to the final submitted manuscript. All authors have reviewed and approved the final version of this manuscript.

Conflict of interest: A.D. Doyle reports grants from NIH (F31HL124959), donor money paid to the Mayo Clinic from Donald R. Levin Family Foundation, and a scholarship at the Mayo Clinic from Mayo Clinic Sidney Luckman Family Predoctoral Fellowship, during the conduct of the study. M. Mukherjee has nothing to disclose. W.E. LeSuer has nothing to disclose. T.B. Bittner has nothing to disclose. S.M. Pasha has nothing to disclose. J.J. Frere has nothing to disclose. J.L. Neely has nothing to disclose. J.A. Kloeber has nothing to disclose. K.P. Shim has nothing to disclose. S.I. Ochkur has nothing to disclose. T. Ho has nothing to disclose. S. Svenningsen has nothing to disclose. B.L. Wright reports donor money paid to the Mayo Clinic from Donald R. Levin Family Foundation, during the conduct of the study. M.A. Rank has nothing to disclose. J.J. Lee received grants from NIH (HL058723), NIH NCRR (K26 RR0109709) and the Mayo Foundation for Medical Education and Research, during the conduct of this study; J.J. Lee is deceased and this statement was made on behalf of the author by the corresponding author. P. Nair reports grants and personal fees for consultancy and lecturing from AstraZeneca and Teva, grants from Boehringer Ingelheim, grants and personal fees for lecturing from Novartis, grants and personal fees for consultancy from Sanofi and GSK, and personal fees for consultancy from Theravance and Knopp, outside the submitted work. E.A. Jacobsen reports grants from NIH (HL065228), and donor money paid to the Mayo Clinic from Donald R. Levin Family Foundation, during the conduct of the study.

Support statement: This work was supported by Mayo Foundation for Medical Education and Research, NIH NHLBI R01 HL058723 and HL065228, and NIH NCRR K26 RR0109709. A.D. Doyle was supported by the Mayo Graduate School, the Mayo Clinic Sidney Luckman Family Predoctoral Fellowship, the Donald R. Levin Family Foundation and 
NIH NHLBI F31HL124959. P. Nair is supported by the Frederick E. Hargreave Teva Innovation Chair in Airway

Diseases. Funding information for this article has been deposited with the Crossref Funder Registry.

\section{References}

1 May SM, Li JT. Burden of chronic obstructive pulmonary disease: healthcare costs and beyond. Allergy Asthma Proc 2015; 36: 4-10

2 Turner AM, Tamasi L, Schleich F, et al. Clinically relevant subgroups in COPD and asthma. Eur Respir Rev 2015 24: $283-298$.

3 Bel EH, Ten Brinke A. New anti-eosinophil drugs for asthma and COPD: targeting the trait! Chest 2017; 152: $1276-1282$.

4 Segal LN, Martinez FJ. Chronic obstructive pulmonary disease subpopulations and phenotyping. J Allergy Clin Immunol 2018; 141: 1961-1971.

5 Barnes PJ. Inflammatory mechanisms in patients with chronic obstructive pulmonary disease. J Allergy Clin Immunol 2016; 138: 16-27.

6 Saha S, Brightling CE. Eosinophilic airway inflammation in COPD. Int J Chron Obstruct Pulmon Dis 2006; 1: 39-47.

7 Bafadhel M, Pavord ID, Russell REK. Eosinophils in COPD: just another biomarker? Lancet Respir Med 2017; 5 747-759.

8 Postma DS, Rabe KF. The asthma-COPD overlap syndrome. N Engl J Med 2015; 373: 1241-1249.

9 Vedel-Krogh S, Nielsen SF, Lange P, et al. Blood eosinophils and exacerbations in chronic obstructive pulmonary disease. The Copenhagen General Population Study. Am J Respir Crit Care Med 2016; 193: 956-974.

10 Siddiqui SH, Guasconi A, Vestbo J, et al. Blood eosinophils: a biomarker of response to extrafine beclomethasone/ formoterol in chronic obstructive pulmonary disease. Am J Respir Crit Care Med 2015; 192: 523-525.

11 Hastie AT, Martinez FJ, Curtis JL, et al. Association of sputum and blood eosinophil concentrations with clinical measures of COPD severity: an analysis of the SPIROMICS cohort. Lancet Respir Med 2017; 5: 956-967.

12 Jacobsen EA, Helmers RA, Lee JJ, et al. The expanding role(s) of eosinophils in health and disease. Blood 2012; 120: $3882-3890$

13 Lee JJ, Dimina D, Macias MP, et al. Defining a link with asthma in mice congenitally deficient in eosinophils. Science 2004; 305: 1773-1776.

14 Ochkur SI, Jacobsen EA, Protheroe CA, et al. Coexpression of IL-5 and eotaxin-2 in mice creates an eosinophil-dependent model of respiratory inflammation with characteristics of severe asthma. J Immunol 2007; 178: 7879-7889.

15 Jacobsen EA, Doyle AD, Colbert DC, et al. Differential activation of airway eosinophils induces IL-13-mediated allergic Th2 pulmonary responses in mice. Allergy 2015; 70: 1148-1159.

16 Jacobsen EA, Ochkur SI, Doyle AD, et al. Lung pathologies in a chronic inflammation mouse model are independent of eosinophil degranulation. Am J Respir Crit Care Med 2017; 195: 1321-1332.

17 Gharib SA, Manicone AM, Parks WC. Matrix metalloproteinases in emphysema. Matrix Biol 2018; 73: 34-51.

18 Hautamaki RD, Kobayashi DK, Senior RM, et al. Requirement for macrophage elastase for cigarette smoke-induced emphysema in mice. Science 1997; 277: 2002-2004.

19 Shaykhiev R, Krause A, Salit J, et al. Smoking-dependent reprogramming of alveolar macrophage polarization: implication for pathogenesis of chronic obstructive pulmonary disease. J Immunol 2009; 183: 2867-2883.

20 Pouladi MA, Robbins CS, Swirski FK, et al. Interleukin-13-dependent expression of matrix metalloproteinase-12 is required for the development of airway eosinophilia in mice. Am J Respir Cell Mol Biol 2004; 30: 84-90.

21 Zheng T, Zhu Z, Wang Z, et al. Inducible targeting of IL-13 to the adult lung causes matrix metalloproteinaseand cathepsin-dependent emphysema. J Clin Invest 2000; 106: 1081-1093.

22 Doyle AD, Jacobsen EA, Ochkur SI, et al. Homologous recombination into the eosinophil peroxidase locus generates a strain of mice expressing Cre recombinase exclusively in eosinophils. J Leukoc Biol 2013; 94: 17-24.

23 Lee NA, McGarry MP, Larson KA, et al. Expression of IL-5 in thymocytes/T cells leads to the development of a massive eosinophilia, extramedullary eosinophilopoiesis, and unique histopathologies. J Immunol 1997; 158: $1332-1344$

24 McKenzie GJ, Emson CL, Bell SE, et al. Impaired development of Th2 cells in IL-13-deficient mice. Immunity 1998; 9: 423-432.

25 Jacobsen EA, Zellner KR, Colbert D, et al. Eosinophils regulate dendritic cells and Th2 pulmonary immune responses following allergen provocation. J Immunol 2011; 187: 6059-6068.

26 Lasbury ME, Durant PJ, Lee $\mathrm{CH}$. Numbers of alveolar macrophages are increased during Pneumocystis pneumonia in mice. J Eukaryot Microbiol 2003; 50: Suppl., 637-638.

27 Ochkur SI, Protheroe CA, Li W, et al. Cys-leukotrienes promote fibrosis in a mouse model of eosinophil-mediated respiratory inflammation. Am J Respir Cell Mol Biol 2013; 49: 1074-1084.

28 Knudsen L, Weibel ER, Gundersen HJ, et al. Assessment of air space size characteristics by intercept (chord) measurement: an accurate and efficient stereological approach. J Appl Physiol 2010; 108: 412-421.

29 Ochkur SI, Kim JD, Protheroe CA, et al. A sensitive high throughput ELISA for human eosinophil peroxidase: a specific assay to quantify eosinophil degranulation from patient-derived sources. J Immunol Methods 2012; 384: $10-20$.

30 Jacobsen EA, Ochkur SI, Pero RS, et al. Allergic pulmonary inflammation in mice is dependent on eosinophil-induced recruitment of effector T cells. J Exp Med 2008; 205: 699-710.

31 Doyle AD, Jacobsen EA, Ochkur SI, et al. Expression of the secondary granule proteins major basic protein 1 (MBP-1) and eosinophil peroxidase (EPX) is required for eosinophilopoiesis in mice. Blood 2013; 122: 781-790.

32 McGarry MP, Protheroe CA, Lee JJ. Mouse Hematology: A Laboratory Manual. Cold Spring Harbor, Cold Spring Harbor Laboratory Press, 2010.

33 Pizzichini E, Pizzichini MM, Efthimiadis A, et al. Measurement of inflammatory indices in induced sputum: effects of selection of sputum to minimize salivary contamination. Eur Respir J 1996; 9: 1174-1180.

34 Vestbo J, Hurd SS, Agustí A, et al. Global strategy for the diagnosis, management, and prevention of chronic obstructive pulmonary disease: GOLD executive summary. Am J Respir Crit Care Med 2013; 187: 347-365. 

progression. Chest 2007; 132: 1733-1740. and -12 in IL-13-induced inflammation and remodeling. I Clin Invest 2002; 110: 463-474.

37 Shapiro SD, Kobayashi DK, Ley TJ. Cloning and characterization of a unique elastolytic metalloproteinase produced by human alveolar macrophages. J Biol Chem 1993; 268: 23824-23829.

38 Boorsma CE, Draijer C, Melgert BN. Macrophage heterogeneity in respiratory diseases. Mediators Inflamm 2013; 2013: 769214.

39 Zhao J, Zhao Y. Interleukin-33 and its receptor in pulmonary inflammatory diseases. Crit Rev Immunol 2015; 35: $451-461$.

40 Stolarski B, Kurowska-Stolarska M, Kewin P, et al. IL-33 exacerbates eosinophil-mediated airway inflammation. J Immunol 2010; 185: 3472-3480.

41 Willebrand R, Voehringer D. IL-33-induced cytokine secretion and survival of mouse eosinophils is promoted by autocrine GM-CSF. PLoS One 2016; 11: e0163751.

42 Schmid-Grendelmeier P, Altznauer F, Fischer B, et al. Eosinophils express functional IL-13 in eosinophilic inflammatory diseases. J Immunol 2002; 169: 1021-1027.

43 Doyle A, McGarry MP, Lee NA, et al. The construction of transgenic and gene knockout/knockin mouse models of human disease. Transgenic Res 2012; 21: 327-349.

44 Voehringer $\mathrm{D}$, $\mathrm{Wu} \mathrm{D}$, Liang $\mathrm{HE}$, et al. Efficient generation of long-distance conditional alleles using recombineering and a dual selection strategy in replicate plates. BMC Biotechnol 2009; 9: 69.

45 Ohno I, Ohtani H, Nitta Y, et al. Eosinophils as a source of matrix metalloproteinase-9 in asthmatic airway inflammation. Am J Respir Cell Mol Biol 1997; 16: 212-219.

46 Lee JJ, Jacobsen EA, McGarry MP, et al. Eosinophils in health and disease: the LIAR hypothesis. Clin Exp Allergy 2010; 40: 563-575.

47 Drake LY, Kita H. Group 2 innate lymphoid cells in the lung. Adv Immunol 2014; 124: 1-16.

48 Wills-Karp M. Immunologic basis of antigen-induced airway hyperresponsiveness. Annu Rev Immunol 1999; 17: 255-281.

49 Lagente V, Le Quement C, Boichot E. Macrophage metalloelastase (MMP-12) as a target for inflammatory respiratory diseases. Expert Opin Ther Targets 2009; 13: 287-295.

50 Lavigne MC, Eppihimer MJ. Cigarette smoke condensate induces MMP-12 gene expression in airway-like epithelia. Biochem Biophys Res Commun 2005; 330: 194-203.

51 Pavord ID, Chanez P, Criner GJ, et al. Mepolizumab for eosinophilic chronic obstructive pulmonary disease. N Engl J Med 2017; 377: 1613-1629.

52 Dasgupta A, Kjarsgaard M, Capaldi D, et al. A pilot randomised clinical trial of mepolizumab in COPD with eosinophilic bronchitis. Eur Respir J 2017; 49: 1602486.

53 Brightling CE, Bleecker ER, Panettieri RA, et al. Benralizumab for chronic obstructive pulmonary disease and sputum eosinophilia: a randomised, double-blind, placebo-controlled, phase 2a study. Lancet Respir Med 2014; 2 : 891-901.

54 Bihlet AR, Karsdal MA, Sand JM, et al. Biomarkers of extracellular matrix turnover are associated with emphysema and eosinophilic-bronchitis in COPD. Respir Res 2017; 18: 22.

55 Toledo-Pons N, Noell G, Jahn A, et al. Bone marrow characterization in COPD: a multi-level network analysis. Respir Res 2018; 19: 118

56 Gelb AF, Yamamoto A, Verbeken EK, et al. Further studies of unsuspected emphysema in nonsmoking patients with asthma with persistent expiratory airflow obstruction. Chest 2018; 153: 618-629.

57 Brightling CE, Woltmann G, Wardlaw AJ, et al. Development of irreversible airflow obstruction in a patient with eosinophilic bronchitis without asthma. Eur Respir J 1999; 14: 1228-1230. 